\title{
Assessment of Printed Patient-Educational Materials for Chronic Kidney Disease
}

\author{
Delphine S. Tuot ${ }^{a}$ Elizabeth Davis ${ }^{b} \quad$ Alexandra Velasquez ${ }^{a, b}$ \\ Tanushree Banerjee $^{b}$ Neil R. Powe ${ }^{b, c}$ \\ ${ }^{a}$ Division of Nephrology and ${ }^{b}$ Department of Medicine, University of California San Francisco, and \\ ${ }^{c}$ Center for Vulnerable Populations at San Francisco General Hospital, San Francisco, Calif., USA
}

\section{Key Words}

Chronic kidney disease $\cdot$ Health literacy $\cdot$ Patient education materials

\begin{abstract}
Background: Awareness of chronic kidney disease (CKD) is suboptimal among patients with CKD, perhaps due to poor readability of patient education materials (PEMs). We reviewed the suitability and readability of common PEMs that focused on 5 content areas: basics of CKD, risk factors for CKD development, risk factors for CKD progression, complications of CKD and self-management strategies to improve kidney health. Methods: Three reviewers (nephrologist, primary care physician, patient) used the Suitability Assessment of Materials to rate PEMs on message content/stimulation of learning, typography, visuals and layout and determined literacy level. Mean ratings were calculated for each PEM by content area and overall (superior = 70-100; adequate $=40-69$; inadequate $=<40$ ). Linear regression was used to determine the impact of literacy level on mean rating. Results: We reviewed 69 PEMs from 19 organizations, divided into 113 content area sections. Most (79\%) PEM sections were 'adequate' (mean rating, 58.3\%). Inclusion of patient-centered content and opportunities for patient interaction were associated with 'superior' ratings. Mean ratings
\end{abstract}

(SD) were similar across content areas: basics of CKD, 58.9\% (9.1); risk factors for CKD development, 57.0\% (12.3); risk factors for CKD progression, 58.5\% (12.0); CKD complications, $62.3 \%$ (15.7), and self-management strategies, $62.2 \%$ (12.3). $\leq 6$ th grade literacy level (vs. $>6$ th grade) was associated with an 11.7 point higher mean rating. Conclusion: Most PEMs for kidney disease were adequate. Outstanding PEMs shared characteristics of patient centeredness, a low literacy level, and patient interaction. Providers should be aware of strengths and limitations of PEMs when educating their patients about CKD.

ㄷ) 2013 S. Karger AG, Basel

\section{Introduction}

Chronic kidney disease (CKD) affects millions of people in the United States [1] and is associated with cardiovascular morbidity and increased mortality at all stages [2]. Strategies to reduce CKD-related complications, including progression to end-stage renal disease (ESRD), and death, such as glycemic control in persons with diabetes, blood pressure control, reduction of proteinuria, avoidance of nephrotoxic substances (such as non-steroidal anti-inflammatory drugs) and diet and lifestyle modifications, are well-known to clinicians [3-5]. Implemen-

\section{KARGER}

E-Mail karger@karger.com

www.karger.com/ajn
(C) 2013 S. Karger AG, Basel

0250-8095/13/0383-0184\$38.00/0
Delphine S. Tuot

San Francisco General Hospital

1001 Potrero Avenue, Bldg 100, Room 342

San Francisco, CA 94110 (USA)

E-Mail Delphine.tuot@ucsf.edu 
tation of these CKD risk modification behaviors requires not only clinician awareness, but also patient understanding and engagement in their health [6].

Published data suggest that $<10 \%$ of individuals with $\mathrm{CKD}$ are aware of their kidney disease, including only $16 \%$ of individuals with complicated CKD $[7,8]$. Beyond general awareness, perceived and objective knowledge of kidney disease are suboptimal among individuals actively receiving nephrology care [9]. National efforts are thus underway to increase patient awareness of kidney disease, including the development of patient educational materials (PEMs) for individuals with CKD and those at high risk of developing CKD [10].

With many printed PEMs now available, it is not clear which ones best provide patients with and at high risk of CKD with adequate health information and empower them to become better custodians of their CKD care. This is a particular concern for individuals with low health literacy, who represent over 35\% of the adult United States population [11] and at least $18 \%$ of the adult CKD population [12]. Previous studies have demonstrated that web-based educational materials for CKD are difficult to understand [13]. As printed materials remain the cornerstone of office-based clinician-initiated educational efforts, understanding which materials maximize patient engagement in healthy behaviors is key to helping clinicians and patients achieve control of CKD risk factors, thereby minimizing CKD progression. The aim of this study was to evaluate the suitability (ease of understanding) and readability (reading level) of common printed PEMs for individuals with and at high risk of CKD, focusing on 5 content areas pertinent to such individuals: basics of CKD, risk factors for CKD development, risk factors for CKD progression, early complications of CKD and selfmanagement strategies to improve kidney health. Results could help clinicians select the PEMs best suited for their patients, leading to more effective CKD education, potentially enhancing patient engagement and overall health.

\section{Methods}

Identification of CKD Patient Education Materials

We identified a large convenience sample of common PEMs using Internet search engines (Google and Yahoo) with the following search terms: 'Chronic kidney disease [CKD] patient education materials', 'CKD patient materials', 'CKD patient education resources', 'CKD patient resources', 'CKD patient education information', 'CKD patient information', 'CKD education materials', 'CKD education resources', 'CKD education information', 'CKD materials', 'CKD resources', and 'CKD information'. Web links that contained 2 or more of the above search terms were identified and searched for PEMs. Non-English materials, materials that were purely web based (could not be downloaded and printed as a pdf, text or word document) or those that contained information solely for patients with ESRD were excluded.

We divided each printed material as appropriate into the following content areas: basics of CKD, risk factors for CKD development, risk factors for CKD progression, early complications of CKD and self-management strategies to improve kidney health. This process enabled us to evaluate whether a discrete section of a PEM would be useful in a particular clinical setting, for example, when discussing diet and exercise (self-management) versus anemia (complication of CKD).

\section{Evaluation}

Suitability of Assessment

We assessed PEM sections with an adapted Suitability Assessment of Materials (SAM) instrument [14]. This instrument was originally developed to evaluate the appropriateness and presentation of printed PEMs and has been adapted and validated for evaluation of health-related PEMs for many diseases including congestive heart failure [15], hypertension [16], and stroke [17]. The adapted SAM used in these analyses consisted of 26 items grouped into 4 different domains: message content (including learning stimulation and cultural suitability), text appearance/typography, visuals/graphics, and layout/design.

Three reviewers (D.S.T., nephrologist; E.D., general internist; J.R., a 54-year-old Caucasian patient followed in primary care with proteinuria and obesity) independently rated each unique PEM section using the SAM (fig. 1). The patient reviewer, who has an early high school education, participates in the patient advisory board for her primary care clinic and volunteered to help with this study after a brief recruitment presentation by one of the authors (D.S.T.). Reviewers scored each SAM item according to the following original rating scheme: 1 (inadequate), 2 (adequate), or 3 (superior). Items deemed not applicable to a given material were not scored. Responses that differed substantially among the three reviewers (i.e. responses included inadequate and superior or yes and no ratings for the same item; $\mathrm{n}=369 / 2,857,12.9 \%$ ) were adjudicated to achieve consensus. Adjudicated domain ratings for each PEM section were calculated with the following formula: total points earned/total possible points in that domain. The maximum rating for each domain was 100 . Ratings between $70-100$ were considered 'superior'; ratings 40-69 were deemed 'adequate', and ratings $<40$ were considered 'inadequate'. An overall rating for each PEM section was calculated by averaging the 4 domain scores.

\section{Readability Assessment}

Reviewers independently determined whether each PEM section was $>$ or $\leq$ a 6 th grade reading level. Given that the average American reads at an 8th grade reading level [14], 6th grade reading level is the recommended benchmark for the readability of educational materials in the United States [18]. Sixth grade reading level was defined by presence of short sentences, use of active voice rather than passive voice and most words having 2 syllables or less, consistent with the Simple Measure of Gobbledygook (SMOG) formula of reading level assessment [19]. Readability of each PEM section was determined by consensus. The final overall PEM readability assessment was an average of each of its sections' readability assessments. 


\section{Message Content}

1. Does the material explain the purpose and benefits from the patient's view?

2. Is the content limited to a few essential main points that the majority of the target population will benefit from?

3. Are behaviors and skills emphasized rather than just facts?

4. Are readers provided with opportunities for small successes? (i.e., given the chance to set goals for themselves and monitor their progress)

5. Are key points reviewed at the end of each section/page?

6. Is the material sensitive to cultural differences?

7. Is the new information placed in the context of patients lives? (i.e., is there a patient story or narrative that helps explain the new information?)

8. Are readers told what they should get from the material and what they can do to improve their health? (i.e., is there a clear introduction to the material?)

9. Is the organization of the paragraphs and sentences conducive to easy reading?

10. Are instructions broken into easy-to-read parts?

11. Is the material interactive (encourage the patients to write, answer questions, ask questions, cut out forms, etc.)?

\section{$\square$ Adequate \\ $\square$ Superior}

\section{Text appearance/typography}

12. Is the font size no smaller than $12-14$ point?

$\square$ Inadequate $\square$ Adequate $\square$ Superior

13. Is easy-to-read font used? (no fancy script or lettering)

$\square$ Inadequate

$\square$ Adequate

$\square$ Superior

14. Are bold and underline used instead or ALL CAPS and $\quad$ Inadequate italics?

$\square$ Adequate

$\square$ Superior

15. Are fonts used to promote easy reading? (Dark fonts on light backgrounds are best)

$\square$ Inadequate

$\square$ Adequate

$\square$ Superior

$\square$ Inadequate

$\square$ Adequate

$\square$ Superior

16. Is sharp contrast and large font used?

\section{Visuals/Graphics}

17. Are there visuals in the document?

$\square \mathrm{No}$

17a. Do the visuals all help communicate the messages in a $\quad$ Inadequate literal manner (no abstract symbols)?

$\square$ Adequate

$\square$ Superior

17b. Are the visuals culturally relevant and sensitive?

$\square$ Inadequate

$\square$ Adequate

$\square$ Superior

17c. Are the visuals easy for readers to follow and understand?

$\square$ Inadequate

$\square$ Adequate

$\square$ Superior

18. Are there pictures of body parts?

$\square$ Yes

$\square$ No

18a. Are internal body parts or small objects shown in context and in a realistic manner? (i.e., do they help the reader understand the material?)

$\square$ Inadequate $\square$ Adequate

$\square$ Superior

19. Are the graphics professional and appropriate for an adult $\square$ Inadequate audience?

$\square$ Adequate

$\square$ Superior

20. Are the graphics free of distracting details that take away from the main idea?

$\square$ Inadequate

$\square$ Adequate

$\square$ Superior

21. Do graphics contribute to the message?

$\square$ Inadequate

$\square$ Adequate

$\square$ Superior

22. Are examples given for any lists, charts, or diaries that readers are supposed to complete?

$\square$ Inadequate

$\square$ Adequate

$\square$ Superior

\section{Layout and design}

23. Is the cover effectively designed? (i.e., is it appealing and does it convey what topic the material will cover?)

24. Are messages organized so they are easy to act on and recall (i.e., text boxes to highlight important points)?

25. Is there a lot of white space (no dense text)?

26. Is the text easy for the eye to follow (bullets, paragraph shape: $40-50$ characters wide, text boxes)? $\square$ Inadequate

$\square$ Adequate

$\square$ Superior

$\square$ Inadequate

$\square$ Adequate

$\square$ Superior

$\square$ Inadequate

$\square$ Adequate

$\square$ Superior

$\square$ Inadequate

$\square$ Adequate

$\square$ Superior

Fig. 1. SAM domains and items. 
Table 1. Characteristics and ratings of CKD PEMs

\begin{tabular}{|c|c|c|c|c|c|c|c|c|c|c|c|c|}
\hline \multirow[t]{2}{*}{ Source } & \multirow[t]{2}{*}{ PEM working title } & \multicolumn{2}{|c|}{$\begin{array}{l}\text { Target } \\
\text { population }\end{array}$} & \multicolumn{2}{|c|}{$\begin{array}{l}\text { Reading } \\
\text { level }\end{array}$} & \multicolumn{5}{|c|}{ Content areas and ratings } & \multirow{2}{*}{$\begin{array}{l}\text { Overall } \\
\text { mean } \\
\text { rating }\end{array}$} & \multirow[t]{2}{*}{$\begin{array}{l}\text { Sym- } \\
\text { bol }\end{array}$} \\
\hline & & $\begin{array}{l}\text { gen- } \\
\text { eral }\end{array}$ & $\begin{array}{l}\text { minor- } \\
\text { ity or } \\
\text { ethnic }\end{array}$ & $\begin{array}{l}\leq 6 \text { th } \\
\text { grade }\end{array}$ & $\begin{array}{l}>6 \text { th } \\
\text { grade }\end{array}$ & $\begin{array}{l}\text { Basics } \\
\text { of CKD }\end{array}$ & $\begin{array}{l}\text { risk factors } \\
\text { for CKD } \\
\text { develop- } \\
\text { ment }\end{array}$ & $\begin{array}{l}\text { risk factors } \\
\text { for CKD } \\
\text { progression }\end{array}$ & $\begin{array}{l}\text { early } \\
\text { compli- } \\
\text { cations } \\
\text { of CKD }\end{array}$ & $\begin{array}{l}\text { self-man- } \\
\text { agement } \\
\text { strategies for } \\
\text { kidney health }\end{array}$ & & \\
\hline $\mathrm{AKF}$ & Causes_risk factors & $*$ & & $*$ & & & 91.7 & & & & 91.7 & $\mathbf{\square}$ \\
\hline Kidney School & Module 15: alternatives & $*$ & & & $*$ & & & 84.7 & & & 84.7 & $\mathbf{\square}$ \\
\hline NKDEP & AA brochure & & $*$ & $*$ & & & 84.0 & & & & 84.0 & $\mathbf{\square}$ \\
\hline Kidney School & Module 6: anemia & $*$ & & $*$ & & & & & 74.9 & 82.3 & 78.6 & $\mathbf{\square}$ \\
\hline $\mathrm{NKF}$ & Anemia & $*$ & & $*$ & & & & & 78.5 & & 78.5 & $\mathbf{\square}$ \\
\hline NKF & Iron and kidney disease & $*$ & & $*$ & & & & & 78.1 & & 78.1 & $\mathbf{\square}$ \\
\hline AAKP & Complications & $*$ & & $*$ & & & & & 75.5 & & 75.5 & $\square$ \\
\hline Kidney School & Module 12: staying active & $*$ & & $*$ & & & & & & 75.4 & 75.4 & $\mathbf{\square}$ \\
\hline $\mathrm{AKF}$ & Living well & $*$ & & $*$ & & 68.5 & & & 82.8 & 73.0 & 74.8 & $\square$ \\
\hline $\mathrm{AKF}$ & Anemia & $*$ & & $*$ & & & & & 74.7 & & 74.7 & $\square$ \\
\hline NKF & Nutrition and CKD & $*$ & & $*$ & & & & & & 73.9 & 73.9 & $\mathbf{\square}$ \\
\hline NKDEP & Explaining CKD test results & $*$ & & $*$ & & 72.9 & & & & & 72.9 & $\square$ \\
\hline NKDEP & What is CKD & $*$ & & & $*$ & 73.1 & & & & 71.4 & 72.3 & $\square$ \\
\hline NKDEP & Nutrition fact sheets & $*$ & & $*$ & & & & & 72.9 & 71.5 & 72.2 & $\square$ \\
\hline NKDEP & CKD and meds & $*$ & & $*$ & & & & & & 71.6 & 71.6 & $\mathbf{\square}$ \\
\hline $\mathrm{AKF}$ & High blood pressure & $*$ & $*$ & $*$ & & & & & & 70.9 & 70.9 & $\mathbf{\square}$ \\
\hline Kidney School & Module 1: basics & $*$ & & $*$ & & 65.8 & 68.3 & & 78.5 & & 70.9 & $\mathbf{\square}$ \\
\hline $\mathrm{AKF}$ & Facts about CKD & $*$ & $*$ & $*$ & & 70.1 & & & & & 70.1 & $\mathbf{\square}$ \\
\hline $\mathrm{AKF}$ & Diabetes & $*$ & & $*$ & & & & & & 70.1 & 70.1 & $\mathbf{\square}$ \\
\hline NKDEP & Nutrition and CKD & $*$ & & $*$ & & & & & & 68.6 & 68.6 & $\mathbf{D}$ \\
\hline NKDEP & Sodium & $*$ & & $*$ & & & & 68.0 & & & 68.0 & $\mathbf{D}$ \\
\hline NKDEP & CKD for those with diabetes or HTN & $*$ & & $*$ & & & 66.5 & & & & 66.5 & $\mathbf{D}$ \\
\hline $\mathrm{NKF}$ & About CKD & $*$ & $*$ & $*$ & & 66.5 & 65.6 & 58.2 & 73.2 & 68.3 & 66.4 & $\mathbf{D}$ \\
\hline NKDEP & CKD - what does it mean & $*$ & & $*$ & & 64.3 & & & & 66.5 & 65.4 & $\mathbf{D}$ \\
\hline $\mathrm{NKF}$ & BP and kidneys & $*$ & $*$ & $*$ & & & 65.3 & & & & 65.3 & $\mathbf{D}$ \\
\hline NKDEP & Healthy eating for CKD & $*$ & & $*$ & & & & & & 64.5 & 64.5 & $\mathbf{D}$ \\
\hline NIDDK & Your kidneys & $*$ & & & $*$ & 62.4 & 65.0 & & 65.6 & & 64.3 & $\mathbf{D}$ \\
\hline NKF & Are you at risk for CKD & $*$ & $*$ & $*$ & & 63.8 & 53.4 & 73.2 & & & 63.5 & $\mathbf{D}$ \\
\hline DCI & Complications & $*$ & & $*$ & & & & & 62.8 & & 62.8 & $\mathbf{D}$ \\
\hline $\mathrm{NKF}$ & Diabetes and CKD & $*$ & & $*$ & & & 65.2 & 60.2 & & & 62.7 & $\mathbf{D}$ \\
\hline AAKP & Kidney beginnings & $*$ & & & $*$ & 63.6 & 59.6 & & & 64.7 & 62.6 & $\mathbf{D}$ \\
\hline $\mathrm{NKF}$ & Info about kidneys & $*$ & $*$ & $*$ & & 52.0 & 66.0 & & & & 59.0 & $\mathbf{D}$ \\
\hline NIDDK & Proteinuria & $*$ & $*$ & & $*$ & 58.8 & & & & & 58.8 & $\mathbf{D}$ \\
\hline Life Options & CKD info & $*$ & & & $*$ & 58.2 & 57.4 & & 47.9 & 71.2 & 58.7 & $\mathbf{D}$ \\
\hline $\mathrm{NKF}$ & Staying fit & $*$ & & & $*$ & & & & & 57.8 & 57.8 & $\mathbf{D}$ \\
\hline Kidney School & Module 13: heart health & $*$ & & $*$ & & & & & & 57.6 & 57.6 & $\mathbf{D}$ \\
\hline NIDDK & high blood pressure & $*$ & $*$ & & $*$ & & & & & 55.1 & 55.1 & $\mathbf{D}$ \\
\hline $\mathrm{AAKP}$ & I heart my kidneys & $*$ & & & $*$ & 54.5 & 56.7 & & & 48.0 & 53.1 & $\mathbf{D}$ \\
\hline KUF & Analgesia & $*$ & & & $*$ & & 52.1 & & & & 52.1 & $\mathbf{D}$ \\
\hline KUF & About kidneys & $*$ & & $*$ & & 54.0 & 49.2 & & & 52.8 & 52.0 & $\mathbf{D}$ \\
\hline NIDDK & Kidneys-diabetes & $*$ & $*$ & & $*$ & 61.3 & 42.5 & & & & 51.9 & 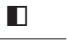 \\
\hline Uptodate & Diabetes & $*$ & & & $*$ & & 51.3 & & & & 51.3 & $\mathbf{D}$ \\
\hline DCI & Strategies & $*$ & & & $*$ & & & & & 51.2 & 51.2 & $\mathbf{D}$ \\
\hline Baxter & What is kidney & $*$ & & & $*$ & 47.2 & & & & 55.1 & 51.2 & $\boldsymbol{D}$ \\
\hline Baxter & Causes of kidney disease & $*$ & & $*$ & & & 51.0 & & & & 51.0 & $\square$ \\
\hline Davita & Progression & $*$ & & & $*$ & & & 51.0 & & & 51.0 & $\mathbf{D}$ \\
\hline
\end{tabular}


Table 1 (continued)

\begin{tabular}{|c|c|c|c|c|c|c|c|c|c|c|c|}
\hline \multirow[t]{2}{*}{ Source } & \multirow[t]{2}{*}{ PEM working title } & $\begin{array}{l}\text { Target } \\
\text { population }\end{array}$ & \multicolumn{2}{|c|}{$\begin{array}{l}\text { Reading } \\
\text { level }\end{array}$} & \multicolumn{5}{|c|}{ Content areas and ratings } & \multirow{2}{*}{$\begin{array}{l}\text { Overall } \\
\text { mean } \\
\text { rating }\end{array}$} & \multirow[t]{2}{*}{$\begin{array}{l}\text { Sym- } \\
\text { bol }\end{array}$} \\
\hline & & $\begin{array}{ll}\text { gen- minor- } & \text { eral ity or } \\
& \text { ethnic }\end{array}$ & $\begin{array}{l}\leq 6 \text { th } \\
\text { grade }\end{array}$ & $\begin{array}{l}>6 \text { th } \\
\text { grade }\end{array}$ & $\begin{array}{l}\text { Basics } \\
\text { of CKD }\end{array}$ & $\begin{array}{l}\text { risk factors } \\
\text { for CKD } \\
\text { develop- } \\
\text { ment }\end{array}$ & $\begin{array}{l}\text { risk factors } \\
\text { for CKD } \\
\text { progression }\end{array}$ & $\begin{array}{l}\text { early } \\
\text { compli- } \\
\text { cations } \\
\text { of CKD }\end{array}$ & $\begin{array}{l}\text { self-man- } \\
\text { agement } \\
\text { strategies for } \\
\text { kidney health }\end{array}$ & & \\
\hline Uptodate & Chronic kidney disease, the basics & $*$ & & $*$ & 51.9 & 49.8 & 50.8 & & 49.8 & 50.6 & $\mathbf{D}$ \\
\hline Fresenius & Diet and nutrition & $*$ & $*$ & & & & & 49.5 & & 49.5 & $\mathbf{D}$ \\
\hline KCP & Kidney disease & $*$ & & $*$ & 49.2 & & & & & 49.2 & $\mathbf{D}$ \\
\hline Fresenius & Healthy kidneys & $*$ & & $*$ & 48.6 & & & & & 48.6 & $\mathbf{D}$ \\
\hline Davita & Self-management & $*$ & & $*$ & & & & & 48.6 & 48.6 & $\mathbf{D}$ \\
\hline$\overline{\mathrm{DCI}}$ & Kidney disease & $*$ & & $*$ & 48.3 & 47.8 & & & & 48.1 & $\mathbf{D}$ \\
\hline Baxter & Diet for CKD & $*$ & $*$ & & & & 48.0 & & & 48.0 & $\mathbf{\square}$ \\
\hline Satellite dialysis & Newly diagnosed & $*$ & $*$ & & 52.3 & 42.1 & & & & 47.2 & $\mathbf{D}$ \\
\hline Uptodate & High cholesterol & $*$ & $*$ & & & & 47.1 & & & 47.1 & D \\
\hline $\mathrm{ADA}$ & Kidney disease & $*$ & & $*$ & & 47.0 & & & & 47.0 & $\square$ \\
\hline Davita & All stages & $*$ & & $*$ & 45.8 & & 47.4 & & & 46.6 & $\mathbf{D}$ \\
\hline Davita & Demographics & $*$ & & $*$ & & 46.5 & & & & 46.5 & $\mathbf{D}$ \\
\hline RAI & CKD and diabetes & $*$ & $*$ & & 50.0 & 42.9 & & & & 46.5 & $\mathbf{D}$ \\
\hline Baxter & Complications of CKD & $*$ & $*$ & & & & & 46.3 & & 46.3 & $\mathbf{D}$ \\
\hline Davita & Understanding & $*$ & & $*$ & 46.3 & & & & & 46.3 & $\square$ \\
\hline Uptodate & Complications & $*$ & $*$ & & & & & 46.2 & & 46.2 & D \\
\hline Davita & Preventing & $*$ & & $*$ & & 45.9 & & & & 45.9 & D \\
\hline ANNA & CKD fact sheet & $*$ & & $*$ & 47.9 & & 42.9 & & & 45.4 & D \\
\hline ANNA & Bone fact sheet & $*$ & & $*$ & & & & 45.1 & & 45.1 & $\mathbf{D}$ \\
\hline Abbott & Improving & $*$ & & $*$ & & & & 43.9 & & 43.9 & $\mathbf{D}$ \\
\hline Davita & Anemia & $*$ & & $*$ & & & & 43.6 & & 43.6 & D \\
\hline Davita & Complications & $*$ & & $*$ & & & & 42.9 & & 42.9 & $\mathbf{D}$ \\
\hline Fresenius & Kidney disease & $*$ & $*$ & & & 42.3 & & & 42.1 & 42.2 & $\mathbf{D}$ \\
\hline
\end{tabular}

NKF = National Kidney Foundation; NKDEP = National Kidney Disease Education Program; NIDDK = National Institute of Diabetes and Digestive and Kidney Disease; AAKP = American Association of Kidney Patients; AKF = American Kidney Fund; ANNA = American Nephrology Nurses Association; KUF $=$ Kidney Urological Foundation; DCI $=$ Dialysis Clinical Incorporated; KCP $=$ Kidney Care Partners; ADA = American Diabetes Association .

$\square$ = Inadequate (0-40\%); $\mathbf{D}=$ adequate ( $41-69 \%) ; \mathbf{\square}=$ superior $(70-100 \%)$.

\section{Statistical Analysis}

Inter-rater reliability for overall PEM ratings using the SAM was determined by intra-class correlation (ICC). Linear regression was used to determine the impact of readability $(>$ or $\leq 6$ th grade reading level) on the mean overall ratings of each PEM section. Stata v11 (StataCorp, College Station, Tex., USA) was used for all analyses.

\section{Results}

\section{Characteristics of PEMs}

We compiled 69 written CKD PEMs created by 19 different organizations. Characteristics of the PEMs, along with the content areas they covered, the average ratings of each content area and the overall mean ratings are delineated in table 1. Twelve (17\%) PEMs targeted African-
American or Latino populations, whereas the remainder targeted the general population. Fourteen (20\%) PEMs were created by governmental organizations (National Kidney Disease Education Program, National Institute of Diabetes and Digestive and Kidney Diseases); 30 (42\%) were produced by kidney disease advocacy groups $(\mathrm{Na}-$ tional Kidney Foundation, American Association of Kidney Patients, American Kidney Fund, American Nephrology Nurses Association, Kidney Care Partners, Life Options, Kidney Urological Foundation, American Diabetes Association and Kidney School), and 25 (36\%) were developed by large dialysis organizations, pharmaceutical companies or information services companies (Fresenius, DaVita, Dialysis Clinical Incorporated, Renal Advantage Incorporated, Satellite Dialysis, Abbott, Baxter, and Uptodate). 
Table 2. Domain ratings for patient educational materials, separated by content area

\begin{tabular}{|c|c|c|c|c|c|c|c|c|}
\hline \multirow[t]{2}{*}{ Content area } & \multirow[t]{2}{*}{$\mathrm{n}$} & \multicolumn{4}{|c|}{ Domain ratings $\pm \mathrm{SD}$} & \multicolumn{3}{|c|}{ Mean domain rating } \\
\hline & & message content & text appearance & visuals & layout & mean $\pm S D$ & $\min$. & $\max$ \\
\hline Risk factors for CKD development & 29 & $57.9 \pm 10.2$ & $65.8 \pm 14.4$ & $49.6 \pm 16.9$ & $55.1 \pm 8.3$ & $57.0 \pm 12.3$ & 42.1 & 91.7 \\
\hline Risk factors for CKD progression & 12 & $61.2 \pm 14.1$ & $74.4 \pm 18.2$ & $46.2 \pm 18.3$ & $53.5 \pm 12.0$ & $58.5 \pm 12.0$ & 42.9 & 84.7 \\
\hline Early complications of CKD & 19 & $60.4 \pm 12.0$ & $80.0 \pm 19.8$ & $50.0 \pm 13.5$ & $59.2 \pm 21.7$ & $62.3 \pm 15.7$ & 42.88 & 82.8 \\
\hline Mean rating & & 60.3 & 70.5 & 51.9 & 57.7 & 58.3 & & \\
\hline
\end{tabular}

Table 3. The top 5 rated PEMs for each CKD content area

\begin{tabular}{|c|c|c|c|c|c|c|c|c|c|c|c|}
\hline \multirow[t]{2}{*}{ Source } & \multirow[t]{2}{*}{ Working title } & \multicolumn{4}{|c|}{ Characteristics } & \multicolumn{4}{|c|}{ Domain ratings, $\%$} & \multirow{2}{*}{$\begin{array}{l}\text { Average } \\
\text { domain } \\
\text { rating, } \\
\%\end{array}$} & \multirow{2}{*}{$\begin{array}{l}\text { Sym- } \\
\text { bol }\end{array}$} \\
\hline & & $\begin{array}{l}\text { gen- } \\
\text { eral }\end{array}$ & $\begin{array}{l}\text { minority } \\
\text { or ethnic }\end{array}$ & $\begin{array}{l}\leq 6 \text { th } \\
\text { grade }\end{array}$ & $\begin{array}{l}>6 \text { th } \\
\text { grade }\end{array}$ & $\begin{array}{l}\text { message } \\
\text { content }\end{array}$ & $\begin{array}{l}\text { text } \\
\text { appear- } \\
\text { ance }\end{array}$ & $\begin{array}{l}\text { visu- } \\
\text { als }\end{array}$ & $\begin{array}{l}\text { lay- } \\
\text { out }\end{array}$ & & \\
\hline \multicolumn{12}{|c|}{ Content area: basics of $C K D$} \\
\hline NKDEP & What is CKD & $*$ & & * & & 57.6 & 93.3 & 66.7 & 75.0 & 73.1 & $\mathbf{\square}$ \\
\hline $\mathrm{AKF}$ & Living well with CKD & $*$ & & * & & 78.8 & 66.7 & 61.9 & 66.7 & 68.5 & $\mathbf{D}$ \\
\hline NKF & About CKD & $*$ & & $*$ & & 57.6 & 66.7 & 66.7 & 75.0 & 66.5 & $\mathbf{D}$ \\
\hline \multicolumn{12}{|c|}{ Content area: risk factors for CKD development } \\
\hline $\mathrm{AKF}$ & Kidney disease: what do I need to know? & $*$ & & * & & 84.8 & 100.0 & 90.5 & 91.7 & 91.7 & - \\
\hline NKDEP & What AA with DM or HTN need to know & & * & * & & 72.8 & 100.0 & 71.4 & 91.7 & 84.0 & a \\
\hline Fresenius & Kidney School: basics of CKD & $*$ & & & $*$ & 73.3 & 66.7 & 66.7 & 66.7 & 68.3 & $\mathbf{D}$ \\
\hline Kidney School & Alternatives for your health & $*$ & & & * & 81.8 & 100.0 & 90.5 & 66.7 & 84.7 & घ \\
\hline $\mathrm{NKF}$ & Are you at risk for CKD & $*$ & * & * & & 60.1 & 93.3 & 55.6 & 83.3 & 73.2 & $\bar{\square}$ \\
\hline NKDEP & Sodium: risk factors for CKD & $*$ & & * & & 63.6 & 100.0 & 58.3 & 50.0 & 68.0 & $\mathbf{D}$ \\
\hline $\mathrm{NKF}$ & Diabetes and CKD & $*$ & & * & & 54.5 & 66.7 & 61.1 & 58.3 & 60.2 & $\mathbf{D}$ \\
\hline NKF & About CKD & $*$ & $*$ & $*$ & & 60.6 & 66.7 & 55.6 & 50.0 & 58.2 & $\mathbf{D}$ \\
\hline \multicolumn{12}{|c|}{ Content area: complications of CKD } \\
\hline AKF & Living well with CKD & $*$ & & $*$ & & 72.7 & 100.0 & 66.7 & 91.7 & 82.8 & - \\
\hline NKF & Anemia and CKD & $*$ & & $*$ & & 66.7 & 100.0 & 55.6 & 91.7 & 78.5 & $\bar{\square}$ \\
\hline Kidney School & Basics of CKD & $*$ & & $*$ & & 84.8 & 100.0 & 55.6 & 75.0 & 78.5 & - \\
\hline NKF & Iron and kidney disease & $*$ & & * & & 63.6 & 100.0 & 57.1 & 91.7 & 78.1 & - \\
\hline AAKP & Complications of CKD & $*$ & & $*$ & & 70.0 & 100.0 & 57.1 & 75.0 & 75.5 & घ \\
\hline \multicolumn{12}{|c|}{ Content area: self-management strategies for kidney health } \\
\hline
\end{tabular}




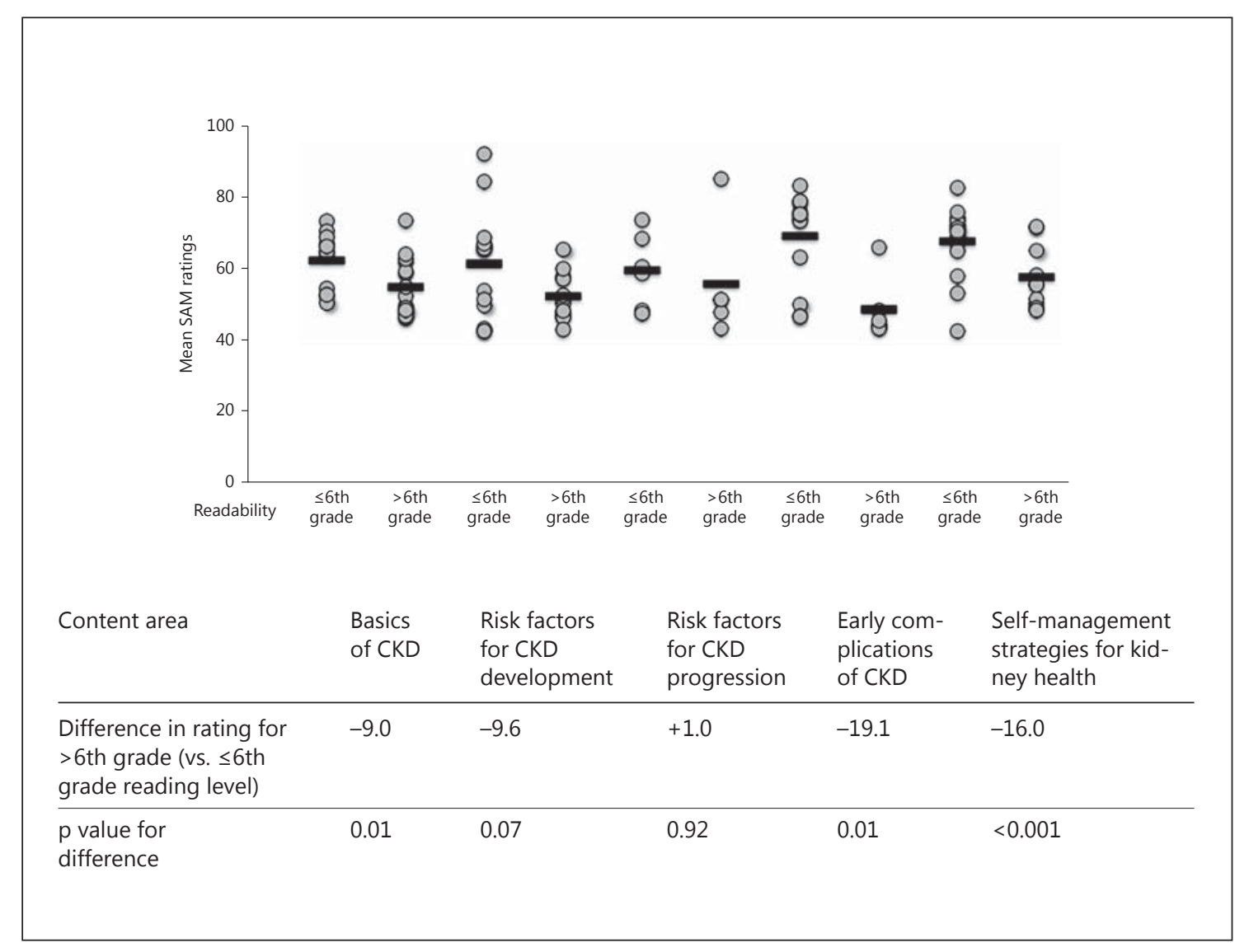

Fig. 2. Association of readability with ratings, by content area. $p$ values for difference in ratings by readability were determined by linear regression.

\section{Inter-Rater Reliability and Ratings of PEMs}

ICC for all 3 reviewers was 0.60 . ICC for the 2 physician raters was 0.75 . This difference was driven primarily by the message content domain of the SAM, for which the patient reviewer rated materials on average 13 points higher than the physician reviewers $(\mathrm{p}<0.001)$.

Using the adjudicated responses, most (79\%) PEM sections were deemed 'adequate' with an overall rating between 40 and 69 . Approximately $20 \%$ of CKD PEM sections were considered 'superior', with an overall rating between 70-100. No CKD PEM section was deemed 'inadequate'. The overall mean PEM rating was 58.3, with modest differences across content areas. Mean ratings were highest for the PEM sections that focused on early complications of CKD (62.3, SD = 15.7); ratings were lowest for the PEM sections that concentrated on risk factors for CKD development $(57.0, \mathrm{SD}=2.3$; table 2). Table 3 illustrates the detailed performance data of the top 5 rated PEMs by content area.
With regard to the domains captured by the SAM items, only the text appearance/typography domain had a mean rating in the 'superior' range (70.5). The other three domains had mean ratings within the 'adequate' range: message content, 60.3 ; visuals/graphics 51.9 , and layout/design, 57.7 (table 2).

\section{Reading Level of PEMs and Impact on Ratings}

Nearly one half of the PEMs (30/69) were determined to have a reading level higher than that of a 6 th grade student. A higher than 6 th grade reading level (compared to $\leq 6$ th grade) was associated with an 11.7-point decrease $(\mathrm{p}<0.01)$ in overall mean PEM ratings. Differences in ratings between PEMs of higher and lower reading levels ranged from +1 point $(p=0.92)$ in the PEM sections that focused on risk factors for CKD progression to -19.1 points ( $p=0.01)$ in the sections that discussed complications of CKD (fig. 2). 


\section{Discussion}

This evaluation of printed English PEMs for patients with CKD demonstrates variable suitability of existing materials, with a mean rating of 58.3/100 and an overall range of $42.2-91.7$, as determined by the validated SAM instrument. While no educational materials were deemed 'inadequate' and all materials were at least considered 'adequate', only $20 \%$ received a 'superior' rating. Ratings (and variability in ratings) of CKD educational materials were comparable across the 5 different content areas that we evaluated. These results are similar to scores that have been published from evaluations of PEMs for other medical conditions, suggesting high availability of health-related PEMs that are decent or good enough, but relatively few that are outstanding [15-17, 20, 21].

Physicians and patients may differ in what they think is important to communicate (and how). In our study, the patient reviewer consistently rated the patient centeredness of the message content more highly than the physician reviewers, with concomitant lower but non-statistically significant differences in ratings of the other SAM domains (typography, visuals and layout/design). Adjudication of results may have thus resulted in lower ratings for the message content domain, suggesting that educational materials may actually convey information more frequently in a patient-centered, culturally appropriate manner than believed by clinicians or depicted in this study. Another explanation could be that the clinician reviewers had higher standards for patient-centered content because of prior exposure to patient-centered PEMs for other diseases. Nevertheless, most PEMs that achieved a 'superior' rating exhibited similar strengths, suggesting that both the patient and clinician reviewers shared similar ideals of what makes an ideal PEM.

One characteristic shared by 'superior' PEMs was providing health information in the context of a patient's experience. One highly rated PEM dedicated to early complications of CKD uses first-person vignettes to present anemia in a patient-centered fashion: 'I learned early that I'm the one who knows my body best. I knew something wasn't right when I constantly felt tired and worn out. I talked to my doctor and found out I was anemic. ... Anemia is explained in the section below.' In contrast, a less highly rated PEM about anemia documented similar symptoms without the accompanying story: 'Anemia happens when your body is not making enough red blood cells. ... Symptoms of anemia can include the following: feeling weak, feeling tired or fatigued, dizziness...'

CKD Patient Educational Materials
Highly rated PEMs also included language, tables, and charts that stimulate readers to interact with the material, encouraging them to participate in their own CKD care. Motivating language in 'superior' PEMs is positive, such as in the following example from a pamphlet that discusses the basics of CKD: 'You may be wondering what you can do now to keep your kidneys as healthy as possible. With early treatment, you may be able to help keep your CKD from getting worse.' By contrast, 'adequate' PEMs use neutral language with a less optimistic feel, such as the following example: 'Have you been told that you have kidney problems or that laboratory tests show your kidney function is not normal? If so, you may be ... at risk for ... kidney failure.' Charts and tables that accompany the optimistic language are essential to help patients visualize and internalize the topics covered. For example, two 'superior' materials encouraged patients to write down questions for their providers and one included a section for action planning with a fill-in-the-blank paragraph.

Inclusion of such patient-centered and behavior change-oriented content is the hallmark of an ideal educational material, and is an important PEM characteristic about which clinicians should be aware. Patient-centered content is of particular importance, as qualitative data suggest that patients want practical and specific information to support their medical knowledge and self-care efforts, rather than general advice from experts [22]. In addition, patient-centered care and patient-centered educational efforts, both central to the Chronic Care Model [23], are associated with improved patient self-efficacy and improved outcomes [24]. Patients also benefit from learning from other patient experiences. For example, provision of an asthma workbook to patients that included narrative vignettes informed by actual patients has been associated with improvement in patient self-efficacy and self-management $[25,26]$. Action planning - making very specific goals and writing them down - is a cornerstone of behavior change science. Studies among patients with chronic diseases have demonstrated that action planning increases the likelihood that patients actually reach their goals [27-29]. PEMs that have incorporated these concepts may be of most clinical utility for patients and providers when discussing CKD.

With respect to graphics, while most of the materials we assessed had visuals/pictures that were deemed professional and easy to understand, they often did not contribute to the overall message. Educational theory suggests that presenting congruent information in multiple formats increases comprehension [30]. The importance of pictures for enhanced patient understanding of the 
risks/benefits of medication treatment [31] and clinical research endeavors [32], as well as comprehension of medical illnesses [33] has been well documented. Graphics can play a particularly important role in shaping patient understanding of kidney disease since the concepts of blood pressure and glomerular filtration can be difficult to explain with words. Providers may find PEMs with pictures and explanatory captions to explain CKD concepts most useful as an adjunct to their discussions with patients.

Approximately one half of the PEMs we evaluated had a reading level at or below that of a 6th grade student. There is considerable evidence that low health literacy is associated with poor health outcomes among patients with chronic diseases [34], including many associated with CKD, such as diabetes $[35,36]$, congestive heart failure [37] and hypertension [38]. More recently, this has been recognized among individuals with ESRD, in whom low health literacy is associated with decreased access to kidney transplantation and increased mortality [39-41]. The relationship between low literacy and adverse health outcomes is likely present among individuals with CKD as well, but it has not yet been extensively explored. Many mechanisms exist by which limited health literacy can influence kidney and overall health, including a negative impact on patient knowledge/beliefs [42]. As an example, a recent study among patients with CKD and diabetes demonstrated a significant and independent association between patient knowledge about their blood pressure goal and achievement of systolic blood pressure $<130 \mathrm{~mm}$ $\mathrm{Hg}$ [43]. In addition to knowledge, health literacy affects other constructs that are key to supporting patient selfcare and improving health outcomes, such as motivation for behavior change, problem-solving ability, and self-efficacy [44]. Use of low-literacy CKD PEMs by clinicians for all patients may not only ensure patient understanding but also increase patient self-efficacy for CKD selfmanagement. Indeed, we found an association between lower reading level and higher PEM ratings, suggesting that materials with shorter sentences/words may communicate their message more clearly, leading to more patient-centered content.

This study is not without its limitations. We did not have information about how printed PEMs were developed or their original intent or purpose. Additionally, we did not evaluate other delivery media such as web-based video or audio. While Internet-based communication is certainly gaining relevance, we focused our evaluation on printed materials that were readily available for providers to give to patients to reinforce verbally delivered CKD education. Printed educational materials have been demonstrated to enhance patient knowledge and understanding of health conditions in randomized controlled trials $[33,45,46]$. They also offer a number of advantages over web-based tools, such as consistency/reliability, portability, and low cost, which maintain their relevancy in an ever-changing world of health communication and education. The SAM is a validated instrument to assess the content and presentation of printed documents but it does not capture all of the important characteristics of a material designed for health education. For example, SAM items do not assess how a reader interacts or uses a given material, the length of the material, the time it takes an individual to read through a PEM, or the number of concepts covered. The SAM also does not include questions that are specifically about kidney disease, and it has not been validated for use by patients. Our analysis, similar to the development of quality measures, systematic reviews and scientific peer review, was based on a small number of well-informed raters, allowing some possibility of bias. However, we were explicit in including a patient rater in our evaluation process, which is novel, and arguably the most important perspective of a PEM's suitability and readability. While the inter-rater reliability among the physicians was consistent or better than that documented by prior studies using the SAM $[15,21]$, the ICC was lower when including patient responses, leading us to adjudicate differences to achieve consensus. Future studies about this topic should be sure to include patients or other end users (i.e. family members, caregivers). Lastly, this analysis does not examine the association of suitability ratings or readability with actual patient understanding, self-efficacy for self-management, or clinical outcomes. Such a study is an essential next step.

The findings from this study have important practice implications. Health care providers are increasingly responsible to empower individuals to participate in their own healthcare. As such, providers charged with the responsibility of educating patients with and at high-risk for CKD need to be aware of strengths and limitations in existing PEMs pertaining to kidney disease. Clinicians could consider preferentially using PEMs written at lower readability levels, as well as those that incorporate patient centeredness, opportunities for patient interaction and motivational language, given our finding that such educational materials achieved higher ratings. This may lead to higher patient awareness of kidney disease and greater patient engagement in CKD self-management, thereby leading to improved health outcomes among individuals with and at risk for CKD. 


\section{Acknowledgements}

This work is supported by the R34DK093992, and Dr. Tuot is supported by K23DK094850, both from the National Institute of Diabetes and Digestive and Kidney Diseases, Bethesda, Md., USA.

\section{Disclosure Statement}

None of the authors have any financial disclosures or conflicts of interest to report.

\section{References}

-1 Coresh J, Byrd-Holt D, Astor BC, Briggs JP, Eggers PW, Lacher DA, Hostetter TH: Chronic kidney disease awareness, prevalence, and trends among U.S. adults, 1999 to 2000. J Am Soc Nephrol 2005;16:180-188.

2 Go AS, Chertow GM, Fan D, McCulloch CE, Hsu CY: Chronic kidney disease and the risks of death, cardiovascular events, and hospitalization. N Engl J Med 2004;351:1296-1305.

3 Barrett BJ: Applying multiple interventions in chronic kidney disease. Semin Dial 2003;16: 157-164.

4 Sarnak MJ, Greene T, Wang X, Beck G, Kusek JW, Collins AJ, Levey AS: The effect of a lower target blood pressure on the progression of kidney disease: long-term follow-up of the modification of diet in renal disease study. Ann Intern Med 2005;142:342-351.

5 Nissenson AR, Collins AJ, Hurley J, Petersen $\mathrm{H}$, Pereira BJ, Steinberg EP: Opportunities for improving the care of patients with chronic renal insufficiency: current practice patterns. J Am Soc Nephrol 2001;12:1713-1720.

-6 Costantini L, Beanlands H, McCay E, Cattran D, Hladunewich M, Francis D: The self-management experience of people with mild to moderate chronic kidney disease. Nephrol Nurs J 2008;35:147-155, quiz 156.

7 Plantinga LC, Tuot DS, Powe NR: Awareness of chronic kidney disease among patients and providers. Adv Chronic Kidney Dis 2010;17: 225-236.

$\checkmark 8$ Tuot DS, Plantinga LC, Hsu CY, Jordan R, Burrows NR, Hedgeman E, Yee J, Saran R, Powe NR: Chronic kidney disease awareness among individuals with clinical markers of kidney dysfunction. Clin J Am Soc Nephrol 2011;6:1838-1844.

-9 Wright Nunes JA, Wallston KA, Eden SK, Shintani AK, Ikizler TA, Cavanaugh KL: Associations among perceived and objective disease knowledge and satisfaction with physician communication in patients with chronic kidney disease. Kidney Int 2011;80:13441351.

10 Hostetter TH, Lising M: National kidney disease education program. J Am Soc Nephrol 2003;14:S114-116.

11 Kutner M, Greenberg E, Jin Y, Paulsen C: The health literacy of America's adults: results from the 2003 national assessment of adult literacy (NCES 2006-483). Washington, National Center for Educational Statistics, US Department of Education, 2006.
12 Fraser SD, Roderick PJ, Casey M, Taal MW Yuen HM, Nutbeam D: Prevalence and associations of limited health literacy in chronic kidney disease: a systematic review. Nephrol Dial Transplant 2013;28:129-137.

13 Calderon JL, Zadshir A, Norris K: Structure and content of chronic kidney disease information on the World Wide Web: barriers to public understanding of a pandemic. Nephrol News Issues 2004;18:76, 78-79, 81-74.

14 Doak CC, Doak LG, Root JH: Teaching Patients with Low Literacy Skills, ed 2. Philadelphia, JB Lippincott, 1996.

15 Taylor-Clarke K, Henry-Okafor Q, Murphy C, Keyes M, Rothman R, Churchwell A, Mensah GA, Sawyer D, Sampson UK: Assessment of commonly available education materials in heart failure clinics. J Cardiovasc Nurs 2012; 27:485-494

16 Lee TW, Kang SJ, Kim HH, Woo SR, Kim S: Suitability and readability assessment of printed educational materials on hypertension (in Korean). J Korean Acad Nurs 2011; 41:333-343.

17 Hoffmann T, Ladner Y: Assessing the suitability of written stroke materials: an evaluation of the interrater reliability of the suitability assessment of materials (SAM) checklist. Top Stroke Rehabil 2012;19:417-422.

18 Kirsch I, Jungeblut A, Jenkins L: Adult literacy in America: a first look at the results of the national adult literacy survey. Washington, Department of Education, 1993.

19 Wang LW, Miller MJ, Schmitt MR, Wen FK: Assessing readability formula differences with written health information materials: application, results, and recommendations. Res Social Adm Pharm 2012, E-pub ahead of print.

20 Fagerlin A, Rovner D, Stableford S, Jentoft C, Wei JT, Holmes-Rovner M: Patient education materials about the treatment of early-stage prostate cancer: a critical review. Ann Intern Med 2004;140:721-728.

21 Vallance JK, Taylor LM, Lavallee C: Suitability and readability assessment of educational print resources related to physical activity: implications and recommendations for practice. Patient Educ Couns 2008;72:342-349.

22 Seligman HK, Wallace AS, DeWalt DA, Schillinger D, Arnold CL, Shilliday BB, Delgadillo A, Bengal N, Davis TC: Facilitating behavior change with low-literacy patient education materials. Am J Health Behav 2007;31(suppl 1):S69-S78.
23 Wagner EH, Austin BT, Davis C, Hindmarsh M, Schaefer J, Bonomi A: Improving chronic illness care: translating evidence into action. Health Aff (Millwood) 2001;20:64-78.

24 Bodenheimer T, Wagner EH, Grumbach K: Improving primary care for patients with chronic illness: the chronic care model. 2. JAMA 2002;288:1909-1914.

25 Mancuso CA, Sayles W, Robbins L, Allegrante JP: Novel use of patient-derived vignettes to foster self-efficacy in an asthma self-management workbook. Health Promot Pract 2010; 11:44-53.

26 Mancuso CA, Sayles W, Allegrante JP: Randomized trial of self-management education in asthmatic patients and effects of depressive symptoms. Ann Allergy Asthma Immunol 2010;105:12-19.

-27 Bischoff EW, Hamd DH, Sedeno M, Benedetti A, Schermer TR, Bernard S, Maltais F, Bourbeau J: Effects of written action plan adherence on COPD exacerbation recovery. Tho$\operatorname{rax} 2011 ; 66: 26-31$.

28 Naik AD, Palmer N, Petersen NJ, Street RL Jr, Rao R, Suarez-Almazor M, Haidet P: Comparative effectiveness of goal setting in diabetes mellitus group clinics: randomized clinical trial. Arch Intern Med 2011;171:453-459.

29 Bodenheimer T, Handley MA: Goal-setting for behavior change in primary care: an exploration and status report. Patient Educ Couns 2009;76:174-180.

30 Paivio A: Mental Representations: A Dual Coding Approach. New York, Clarendon Press, 1986

- 31 Hawley ST, Zikmund-Fisher B, Ubel P, Jancovic $A$, Lucas T, Fagerlin A: The impact of the format of graphical presentation on healthrelated knowledge and treatment choices. $\mathrm{Pa}$ tient Educ Couns 2008;73:448-455.

32 Tait AR, Voepel-Lewis T, Zikmund-Fisher BJ, Fagerlin A: The effect of format on parents' understanding of the risks and benefits of clinical research: a comparison between text, tables, and graphics. J Health Commun 2010; 15:487-501.

-33 You WB, Wolf MS, Bailey SC, Grobman WA Improving patient understanding of preeclampsia: a randomized controlled trial. Am J Obstet Gynecol 2012;206:431 e431-e435.

- 34 Berkman ND, Sheridan SL, Donahue KE Halpern DJ, Crotty K: Low health literacy and health outcomes: an updated systematic review. Ann Intern Med 2011;155:97-107. 
35 Schillinger D, Barton LR, Karter AJ, Wang F, Adler N: Does literacy mediate the relationship between education and health outcomes? A study of a low-income population with diabetes. Public Health Rep 2006;121:245-254.

36 Schillinger D, Grumbach K, Piette J, Wang F, Osmond D, Daher C, Palacios J, Sullivan GD, Bindman AB: Association of health literacy with diabetes outcomes. JAMA 2002;288: 475-482.

37 Peterson PN, Shetterly SM, Clarke CL, Bekelman DB, Chan PS, Allen LA, Matlock DD, Magid DJ, Masoudi FA: Health literacy and outcomes among patients with heart failure. JAMA 2011;305:1695-1701.

-38 Williams MV, Baker DW, Parker RM, Nurss JR: Relationship of functional health literacy to patients' knowledge of their chronic disease. A study of patients with hypertension and diabetes. Arch Intern Med 1998;158:166172.
9 Cavanaugh KL, Wingard RL, Hakim RM, Eden S, Shintani A, Wallston KA, Huizinga MM, Elasy TA, Rothman RL, Ikizler TA: Low health literacy associates with increased mortality in ESRD. J Am Soc Nephrol 2010;21: 1979-1985.

40 Grubbs V, Gregorich SE, Perez-Stable EJ, Hsu CY: Health literacy and access to kidney transplantation. Clin J Am Soc Nephrol 2009; 4:195-200.

41 Green JA, Mor MK, Shields AM, Sevick MA, Arnold RM, Palevsky PM, Fine MJ, Weisbord SD: Associations of health literacy with dialysis adherence and health resource utilization in patients receiving maintenance hemodialysis. Am J Kidney Dis 2013;62:73-80.

42 Osborn CY, Paasche-Orlow MK, Bailey SC, Wolf MS: The mechanisms linking health literacy to behavior and health status. Am J Health Behav 2011;35:118-128.
3 Wright-Nunes JA, Luther JM, Ikizler TA, Cavanaugh KL: Patient knowledge of blood pressure target is associated with improved blood pressure control in chronic kidney disease. Patient Educ Couns 2012;88:184-188.

44 Bodenheimer T, Lorig K, Holman H, Grumbach K: Patient self-management of chronic disease in primary care. JAMA 2002;288: 2469-2475.

45 Partin MR, Nelson D, Radosevich D, Nugent S, Flood AB, Dillon N, Holtzman J, Haas M, Wilt TJ: Randomized trial examining the effect of two prostate cancer screening educational interventions on patient knowledge, preferences, and behaviors. J Gen Intern Med 2004; 19:835-842.

46 Muthusamy AD, Leuthner S, Gaebler-Uhing C, Hoffmann RG, Li SH, Basir MA: Supplemental written information improves prenatal counseling: a randomized trial. Pediatrics 2012;129:e1269-e1274. 